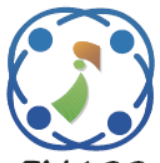

\title{
Detection of Macro and Micro Nodule Using Online Region Based-Active Contour Model in Histopathological Liver Cirrhosis
}

\author{
Nirmala Sapthagiri Guptha ${ }^{1^{*}}$ \\ Kiran Kumari Patil ${ }^{1}$ \\ ${ }^{I}$ Department of Computer Science Engineering, REVA University, India \\ * Corresponding author's Email: avi007surya @gmail.com
}

\begin{abstract}
Automated image examination of histopathology specimens helps for early detection and better characterization of liver cirrhosis. A new method for the segmentation of overlapping nuclei and non-nuclei cell is evaluated, which is named as Online Region based-Active Contour Model (OR-ACM). In this study, a semantic feature extraction (combination of Histogram of Oriented Gradients (HOG) (descriptor level feature) and colour moments (low-level features)) is performed on segmented nuclei cells to evaluate the growth of Endothelial Cells (ECs). It is characterized into two levels such as micro and macro cells that is classified by utilizing Multi-Support Vector Machine (M-SVM). Experimental outcome shows that the proposed approach improved accuracy in liver cirrhosis prediction up to $3 \%$ compared to the existing methods: Adaptively Regularized Kernel-Based Fuzzy C-Means (ARKFCM) Euclidean distance, ARKFCM- Earth movers.
\end{abstract}

Keywords: Active contour model, Endothelial cell, Histogram of oriented gradients, Multi-support vector machine.

\section{Introduction}

In recent years, the digital atlases of human anatomy has become more attention and also reviewed a lot in the area of medical image analysis research [1]. In human, due to haemochromatosis (excess of iron content) cause several diseases like diabetes mellitus, liver cirrhosis and melanoderma, with iron over-load parenchymal and reticuloendothelial cells in numerous organ structures [2]. Among these defects, cirrhosis of liver is one of the growing disease in the medical field [3]. The mechanism behind liver cirrhosis is the ECs, which develops the barrier between the blood stream and wall of the blood vessels and it defends the liver from injury [4]. ECs plays a dynamic role in the regulation of inflammation, vasodilatation, and coagulation stability [5], [6]. Usually, the liver cirrhosis is determined by analysing and reviewing the granular design of liver parenchyma and also illustrated the concerns of outer surface of the liver like ununiformity and roughness [7], [8]. Lot of imaging techniques are available, to evaluate the features of medical images like ultrasound imaging, CT scan imaging etc. [9].

In this research, liver histopathological image is taken as the initial input image for segmentation and classification, which is performed based on their range, texture pattern and structure. Generally, histopathological images include sample of tissues, which significantly studies the sample of free cells and tissue fragments [10]. Also, it returns a more comprehensive view of the disease due to its high resolution. Here, a non-over lapping detection algorithm (OR-ACM) has been implemented to distinguish the normal cells and cirrhotic cells present in the liver, which is based on nucleus separation. Compared to traditional ACMs, the OR-ACM has the advantage of providing less execution time without expression changing. After segmentation, feature extraction is performed using $\mathrm{HOG}$ and colour moments, the combination of both low and high level features obtains optimal feature values. The obtained feature values are given as the input for multiobjective classifier: M-SVM. By utilizing the MSVM classification scheme, the cell system is examined and characterized into nuclei and non- 
nuclei cells. Predicted individual cirrhotic cells are analysed to estimate the level of growth, based on semantic feature extraction. Commonly, the growth of the infected nuclei cell is partitioned into two levels such as Micro module and Macro module. Finally, the experimental outcome confirms that the proposed approach delivers better classification outcome compared to existing approaches.

This paper is organized as follows. Section II survey several recent papers on liver cirrhosis detection. In section III, OR-ACM method is presented with semantic feature extraction and multiobjective classification. In Section IV, the experimental analysis of proposed and existing algorithms are assessed. The conclusion is made in the Section V.

\section{Literature review}

Several researches are suggested by researchers in liver cirrhosis prediction. In this scenario, a brief evaluation of some important contributions to the existing literatures is presented.

A. Chattopadhyay, S. Podder, S. Agarwal, and S. Bhattacharya, [11] have evidently specify the study of Fluoride (F)-induced hepatotoxicity and nephrotoxity in mice by analysing the histopathological changes in the liver, kidney and oxidative stress. To obtain an effective inducer, the Heat Shock Protein (HSP70) in kidney and the liver is negatively regulated in mouse. This specific tissue changes in the HSP synthesis might give helpful biochemical fingerprints or toxicological signature to characterize $\mathrm{F}$ toxicity in human. This proposed technique was less suitable for recognizing the inclined histopathology images, because it took high response time and identification rate.

S. Saleki, A.A. Ardalan, and A.J. Nejad, [12] have demonstrated a major histopathologic finding of phosphine poisoning in the liver, which was determined as the fine cytoplasmic vacuolization of hepatocytes and sinusoidal congestion. The sinusoidal congestion was an intense that cause clumping of red blood cells and the fine cytoplasmic vacuoles is the most consistent finding of their uniform size and shape. Here, the experimental study represented the hepatic histopathologic changes in fatal cases of phosphine poisoning in a large number of cases. The histopathologic evaluation was feasible in all cases. In low resolution dataset, the developed features failed to achieve better recognition by means of accuracy.

G.T. Brown, and D.E. Kleiner, [13] have illustrate the study of Non-alcoholic fatty liver disease (NAFLD) and Non-alcoholic steatohepatitis
(NASH). NAFLD was the critical liver sickness and the NASH was considering as the specific injury pattern in NAFLD. To distinguish the histological changes in NAFLD, staging and grading structures were developed. Results mainly have three grades such as mild, moderate and sever categories based on histologic features. In the first grade, all the features were minimally present and inflammatory components are very mind. In second stage, features were in any degree, but occasionally presented and in the last grade, numerous features were presented. The disadvantage of the proposed methodology was more complex to find the projection of free space. Free space can able to provide better discriminant ability in feature extraction.

M. Ajdari, and M.Z. Ghahnavieh, [14] have investigate the histopathology effects of Nickel Nano-particles (NiNPs) on the lungs, liver and spleen tissues in male mice. The histopathologic investigation result showed that the low to high abnormalities in the liver, spleen, and lung tissues were treated in mice by employing Ni-NPs. Metabolizing the both xenobiotics and vulnerabilities with several reactions may affect the body function of mice. While performing with semi supervised methodologies, the semantic gap was maximized between the feature values, which leads to poor classification rate.

I. Ashok, D. Wankhar, R. Sheeladevi, and W. Wankhar, [15] have specify the study of Long term effect of aspartame on the liver antioxidant status and histopathological in Winstar albino rats. The long term basis might influence the liver and brain, because of its methanol or aspartame, which act as a chemical stressor to alter the antioxidant status and histological pattern. This investigation showed that, while inducing aspartame, it might cause oxidative stress and hepato-renal toxicity. In a few cases, the training phase was semi-automated or manual adjustment, which need to be fully automatic.

To overcome the above mentioned drawbacks, a multi-objective classifier along with the combination of HOG and colour moment is implemented for enhancing the performance of liver cirrhosis prediction.

\section{Proposed methodology}

In this analysis, the OR-ACM scheme is presented to develop the clustering accuracy of cell segmentation. Initially, the region based ACM with Selective Binary and Gaussian Filtering Regularized Level Set (SBGFRLS) is employed as a user-defined function, which is continuously updated by Signed 
Pressure Function (SPF) equation. In mathematically, the SPF equation is specified in Eq. (1):

$$
\operatorname{spf}(I(x, y))=\frac{I(x, y)-\frac{c 1+c 2}{2}}{\max \left(\left|I(x, y)-\frac{c 1+c 2}{2}\right|\right)}
$$

Where, $C 1$ and $C 2$ are determined as constants, and $I(x, y)$ is specified as the input image.

Here, the SPF function regulates the pressure force inside and outside of the Region of Interest (ROI). It will be act as a contour shrinks when outside the object, or enlarges when inside the object. Also, the ACM with SBGFRLS utilizes the consistent variation level set formulation that is symbolized in Eq. (2),

$$
\frac{\partial \varphi}{\partial(x, y)}=\operatorname{spf}(I(x, y)) \times \alpha \times|\nabla \varphi|
$$

Where, $\alpha$ is represented as a constant and speed controller level set and $|\nabla \varphi|$ stated as the gradient of the level set function.

The major hindrance of the ACM with SBGFRLS is that it must be tuned based on the images. However, the tuning procedure is impossible or slowness in high resolution images and real-time video images. In order to overcome these concerns, a modification is done on the ACM, which is named as OR-ACM.

OR-ACM is a region based active contour technique, which does not require any parameters and also the segmentation accuracy is very significant than compared to traditional ACMs schemes. In each iteration, OR-ACM performs a sort of block thresholding procedure. Respective, thresholding procedure generates a rigid boundaries and several minor particles. To eliminate these particles and also to attain smooth and proper object contour, an effective morphological operation is employed.

As similar to ACM with SBGFRLS, the ORACM is also utilizes a user-defined active contour at the initialization step and then constantly updates it. Level set function $\varphi(x, y)$ has different sign symbols such as -1 and +1 , which represents inside and outside of the contour. Unlike the ACM with SBGFRLS, OR-ACM utilizes a simple and significant level updating formulation, which is specified in Eq. (3).

$$
\frac{\partial \varphi}{\partial(x, y)}=H(\operatorname{spf}(I(x, y))) \times \varphi(x, y)
$$

Where $H($.$) is determined as the Heaviside$ function, $I(x, y)$ is stated as an input image and $\varphi(x, y)$ denotes the current level set.
Advantage of OR-ACM over ACM, where OR$\mathrm{ACM}$ does not need any parameter to tune the input images. The mathematical equation of OR-ACM is very easy to understand, unlike other ACM schemes. Therefore, Eq. (3) improves the current level set without affecting the effectiveness of the algorithm. Major variation between OR-ACM and other ACM scheme is smoothing step of level set function. In OR-ACM, a simple morphological opening and closing procedure is followed instead of Gaussian smoothing. After updating procedure of level set, numerous tiny particles are occurred, which are not belong to the object. All these particles can be removed by the opening procedure and the smooth boundaries of objects can be attained by closing.

After the analysis of OR-ACM approach, a watershed algorithm is employed on the output image of OR-ACM. It helps to eliminate the un-wanted particles apart from nuclei cells and non-nuclei cell regions. Also, the overlapped region of nuclei and non-nuclei regions are easily predicted.

\subsection{Semantic feature extraction}

In this scenario, the output images of watershed algorithm is given as the input for semantic feature descriptor (combination of HOG and colour moments). From the histopathology image, the visual features are extracted by applying HOG feature descriptor and colour moment is a low level feature utilized to segment the image, which is based on colour description. Analysis about semantic feature extraction is mentioned below.

\subsubsection{Histogram of oriented gradients (HOG)}

HOG descriptor is a local feature descriptor, which is used for detecting the object. A key characteristic of HOG feature is capability to capture the local appearance of objects, also to account the invariance in object transformations and illumination condition. Since, the edge and information about gradients are determined by applying HOG feature vector. At first, a gradient operator $N$ is employed to calculate the gradient value. The gradient point of the image is denoted by $(x, y)$ and the image frames are expressed as $I$.

$$
G_{x}=N \times I(x, y) \text { and } G_{y}=N^{T} \times I(x, y)
$$

Image detection windows are characterized into various minor spatial regions, which is known as cells. Hence, the magnitude gradients of the pixels are experienced with edge orientation. Finally, the magnitude of the gradients $(x, y)$ is denoted in Eq. (5). 


$$
G_{x}(x, y)=\sqrt{G_{x}(x, y)^{2}}+\sqrt{G_{y}(x, y)^{2}}
$$

Edge orientation of the point $(x, y)$ is specified in Eq. (6).

$$
\theta(x, y)=\tan ^{-1} \frac{G_{y}(x, y)}{G_{x}(x, y)}
$$

Where, $G_{x}$ is mentioned as the horizontal direction of gradients and $G_{y}$ is represented as the vertical direction of gradients.

For superior invariance in illumination and noise, a normalization procedure is performed after the calculation of histogram values. Calculation of normalization is helpful for contrast and also measurements of local histograms are verified. In HOG, four different patterns of normalizations are employed such as L2-norm, L2-Hys, L1-Sqrt and L1norm. Among these normalization L2-norm gives superior performance in cell detection. Blocks of normalization in HOG is represented in Eq. (7),

$$
L_{2-n o r m}: f=\frac{h}{\sqrt{\|h\|_{2}^{2}+e^{2}}}
$$

Where, $e$ is mentioned as a small positive value applied for some regularization, $f$ is mentioned as an extracted feature vector, $h$ is characterised as nonnormalized vector, $\|h\|_{2}^{2}$ is known as 2-norm of HOG normalization.

\subsubsection{Colour moments}

Colour moments (low-level feature) are utilized to distinguish the images based on their colour distribution. Commonly, colour moments depends on three central moments such as, Mean, Standard deviation and Skewness to symbolize the images by using their colour distribution. In this study, these three colour moments are used in this application, because low-order moments accommodate best of the colour information. Respective, three colour moments are expressed as $E_{x}, \sigma_{x}, S_{x}$, which are determined by employing Eqs. (8), (9) and (10).

$$
\begin{aligned}
& E_{x}=\sum_{y-1}^{n} 1 / n^{p_{x y j}} \\
& \sigma_{x}=\sqrt{\left(1 / n \sum_{y-1}^{n}\left(p_{x y}-E_{x}\right)^{2}\right)} \\
& S_{x}=\sqrt[3]{\left(1 / n \sum_{y-1}^{n}\left(p_{x y}-E_{x}\right)^{3}\right)}
\end{aligned}
$$

Where, $p_{x y}$ is represented as the colour value of the $y-t h$ image pixel from $x-t h$ channel, $n$ is specified as the number of pixels in the image, $E_{x}$ gives the average value of the $x-t h$ channel, $\sigma_{x}$ and $S_{x}$ are illustrated as the variance and the skewness of the $x-t h$ channel respectively.

\subsection{Classification method}

In classification section, semantic features are utilized to categorize the images by applying the classifier M-SVM. In this examination, several classes are needed to distinguish (non-nuclei cells, nuclei cells (micro-nuclei cells, macro-nuclei cells)). M-SVM classifier combines multiple-binary classes into single objective function, and also performs significant associated to other classifiers like $\mathrm{K}$ nearest neighbour. Brief description about the MSVM classifier is given below.

\subsubsection{Multi-support vector machine (M-SVM)}

Regular SVMs are basically a two-class classifier, to extend normal SVMs to multi classification, it is essential to modify the multi binary classification issues. Presently, for the Conventional-SVM (C$\mathrm{SVM}$ ), the multi-class classification is rehabilitated into $n-t h$ two-class and for the $i-t h$ two-class issues, class $i$ is distinct from the residual classes.

Two of the prominent methodologies named as One-Against-One (1-a-1) and One-Against-All (1-aa). Here, the 1-a-a solution constructs a binary classifier for each class, which distinct the objects belongs to the class. In $n$ class, the 1-a-a method will generate a $n$ binary classifiers, and the $i-$ th classifier is trained with the samples in $i-$ th class with positive labels and all the remaining samples are trained with negative labels. Final outcome of the $n 1-\mathrm{a}-\mathrm{a}$ classifier correlates to the classifier with the maximum output value. Additionally, the 1-a-1 scheme is resultant from previous research on two class classifiers. The idea of this scheme is to generate all possible two class classifiers from a training set of $n$ classes, each classifier trained only two out of $n$ classes, and there would be $n \times(n-1) / 2$ classifiers.

An effective way to diminish the multi-class issues is based on the decision function, which is constructed by assuming all classes. This technique is an extension to the SVM of medical image classification, to reduce multi-class issues in one step. It is represented in Eq. (11).

$$
\underset{c \sum_{i=1}^{l} \sum_{m \neq y i} \xi_{i}^{m}}{\min \phi(w, \xi)=1 / 2 \sum_{m-1}^{k}\left(w_{m} \cdot w_{m}\right)+}
$$


Subjected to,

$$
\begin{aligned}
& \left(w_{y i} \cdot x i\right)+b_{y i} \geq\left(w_{y i} \cdot x i\right)+b_{m}+2-\xi_{i}^{m} \\
& \xi_{i}^{m} \geq 0, i=1,2, \ldots l, m, y i \epsilon\{1,2, . . k\}, m \neq y i
\end{aligned}
$$

Where, $c$ is a user specified positive constant, $\xi_{i}^{m}$ is mentioned as slack variables, $k$ is specified as the number of classes, $l$ is deliberated as training data point, each $y i$ is the class of the corresponding training data vectors $x i$. Finally, the decision function is assumed using Eq. (14).

$$
f(x)=\operatorname{argmax}\left[\left(w_{i} \cdot x\right)+b_{i}\right], i=1, \ldots k
$$

\section{Experimental results and discussion}

In this scenario, the proposed OR-ACM is examined and processed by employing histopathological image, which is taken from university of UTAH SPENCER S. ECCLES (HEALTH SCIENCES LIBRARY), represents cell based cirrhotic growth of nuclei cells. In classification comparison, numerous histopathological features are utilized in that an active image is selected and the experiment is executed.

\subsection{Experimental setup}

Generally, the histopathology images are high resolution images, which are cropped at the size of $32 * 32$. Histopathology image utilized for this experiment is taken from the database of MICROSCOPY $U$ that gives the essential information about optical microscopy, digital imaging and photomicrography. Sample histopathology image of liver is showed in Fig. 1, which is given as the input image for enhancement or pre-processing step.

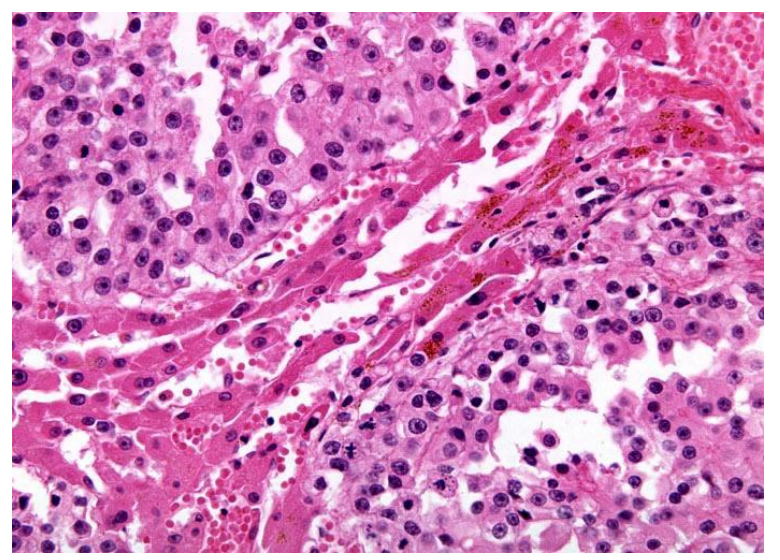

Figure. 1 Input image

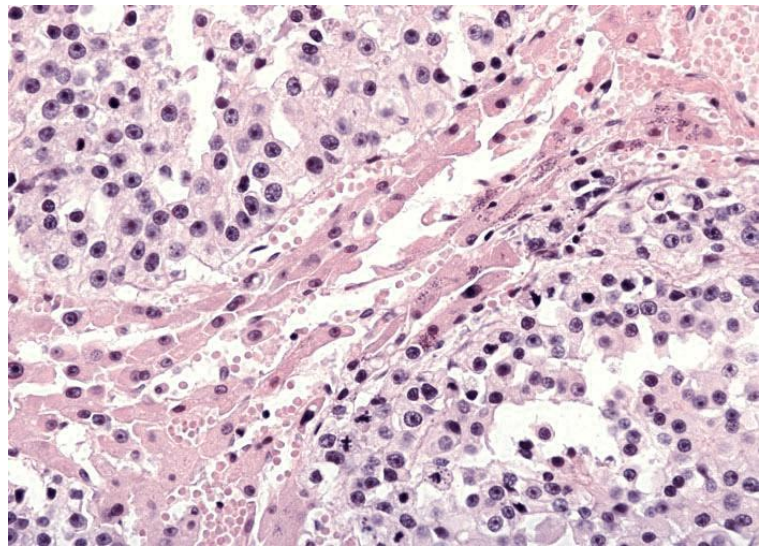

Figure. 2 Enhanced liver image

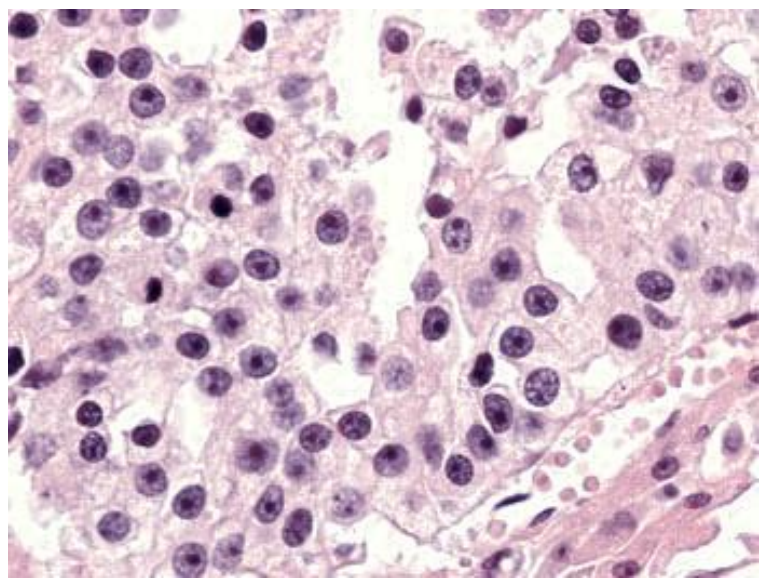

Figure. 3 Region of interest (ROI) from enhanced image

Pre-processed or enhanced histopathology image of liver cirrhosis is specified in Fig. 2. Here, the preprocessing is performed by applying a suitable colour normalization technique. Fig. 3 represents the ROI of enhanced liver image, which is undertaken for further process like segmentation, watershed algorithm etc.

Initially, the image patches are removed from the weakly affected images of training set. In the testing step, extract the image patches cantered at each pixel of the testing image and then employ the superior OR-ACM technique to those patches for generating the probability output labels.

\subsection{Analysis of existing segmentation algorithm}

Existing, Adaptive Regularized Kernel based Fuzzy Clustering Means (ARKFCM) is implemented for extracting the nuclei and non-nuclei images of Fig. 3 (Histopathological ROI image). In ARKFCM, the result occurred in two sets of images, both the images are examined for generating the clustering cells, which are specified in Figs. 4 and 5. The combination of both set of images displayed in Fig. 6 . 


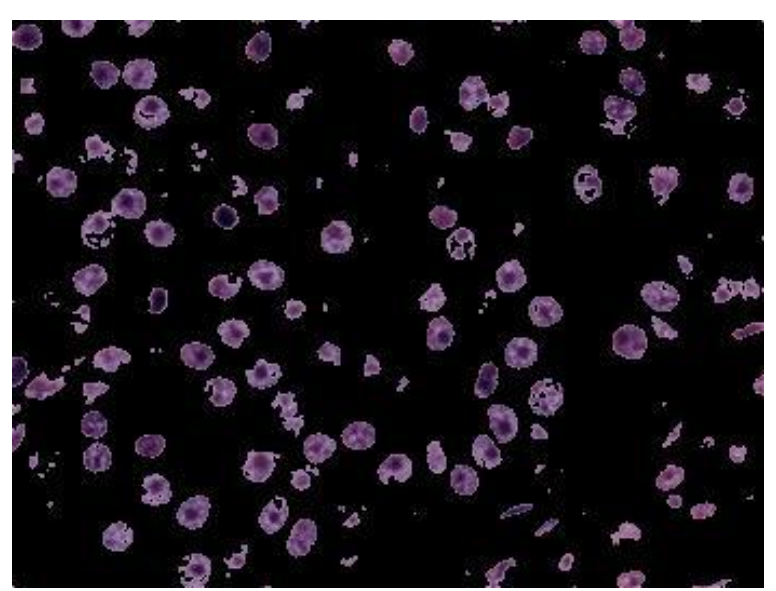

Figure. 4 AFRKFCM first image set

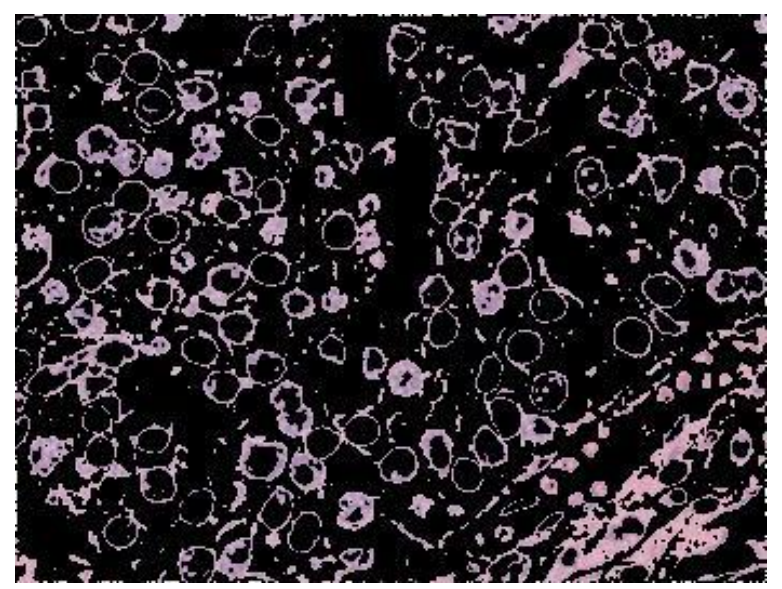

Figure. 5 AFRKFCM second image set

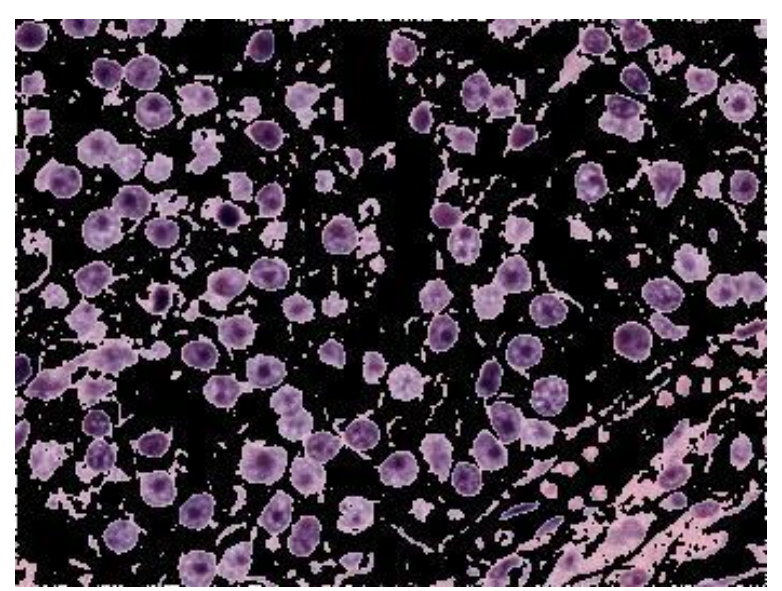

Figure 6 Combined ARKFCM image

Fig. 6 proves that the combined ARKFCM image contains several overlapping regions of cells. Here, the overlapping states (combination of nuclei and non-nuclei cells), is essential to separate those cells to determine the affected cells of liver cirrhosis.

\subsection{Proposed OR-ACM analysis}

In this section, the proposed OR-ACM is employed for segmenting the nuclei cells and nonnuclei cells. OR-ACM is an iteration based approach.
The number of iteration depends on the position of particles (cells). Fig. 7 represented as OR-ACM 1st iterated image and Fig. 8 states 10th iterated image. While comparing this two images, Fig. 8 evidently shows the improvement of OR-ACM in segmentation part.

For better distinguish of overlapped nuclei cells and non-nuclei cells, a watershed algorithm is employed in Fig. 8. Advantage of watershed algorithm is signified in Figs. 9 and 10.

Thereby, nuclei cells, non-nuclei cells, and separated overlapped cells are combined in a single histopathology image, which is symbolized in Fig. 11. Semantic feature extraction is performed in Fig. 11 for better differentiation of nuclei cells and nonnuclei cells.

Further, the non-nuclei and nuclei cells are cropped from OR-ACM image for easy representation, which is stated in Figs. 12 and 13 respectively.

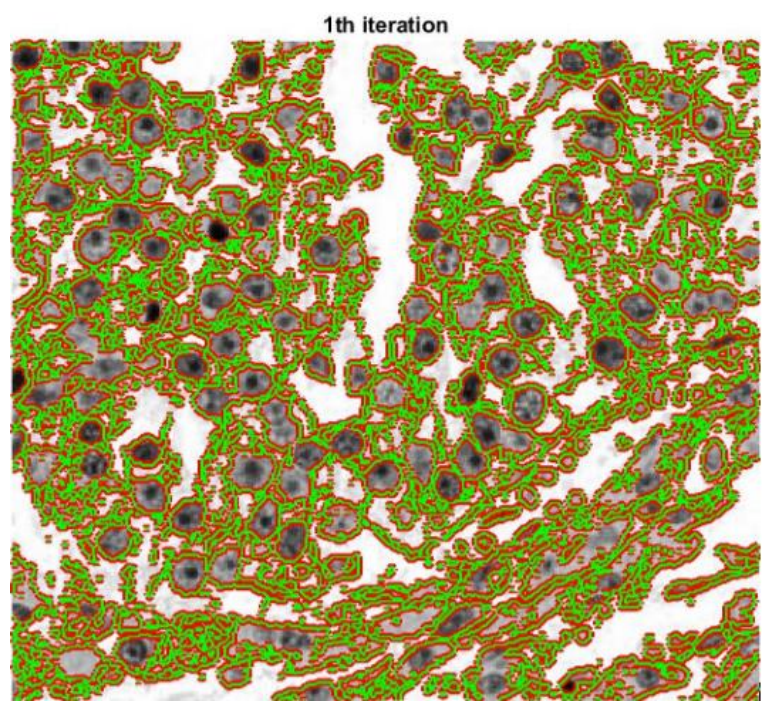

Figure. 7 OR-ACM image for 1st iteration

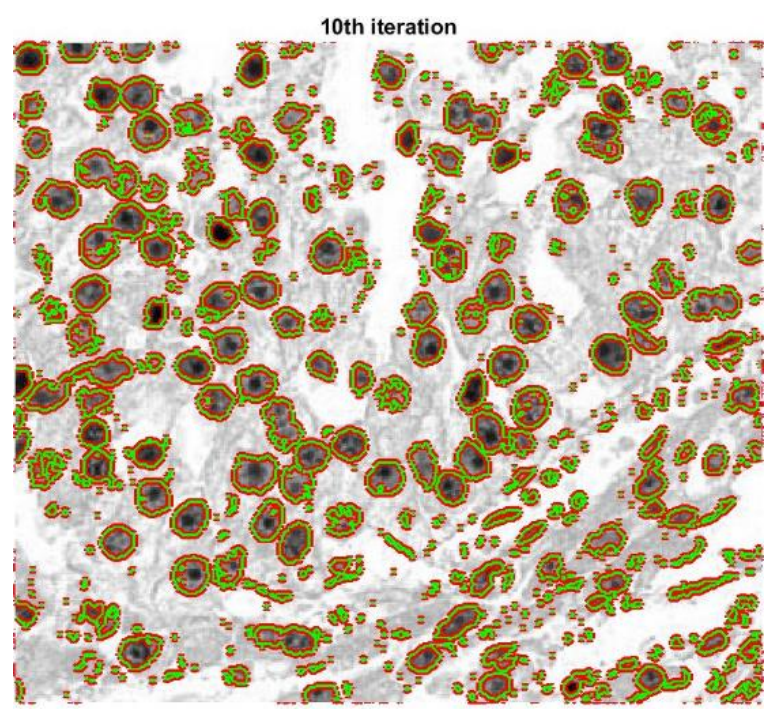

Figure. 8 OR-ACM image for 10th iteration International Journal of Intelligent Engineering and Systems, Vol.11, No.2, $2018 \quad$ DOI: 10.22266/ijies2018.0430.28 


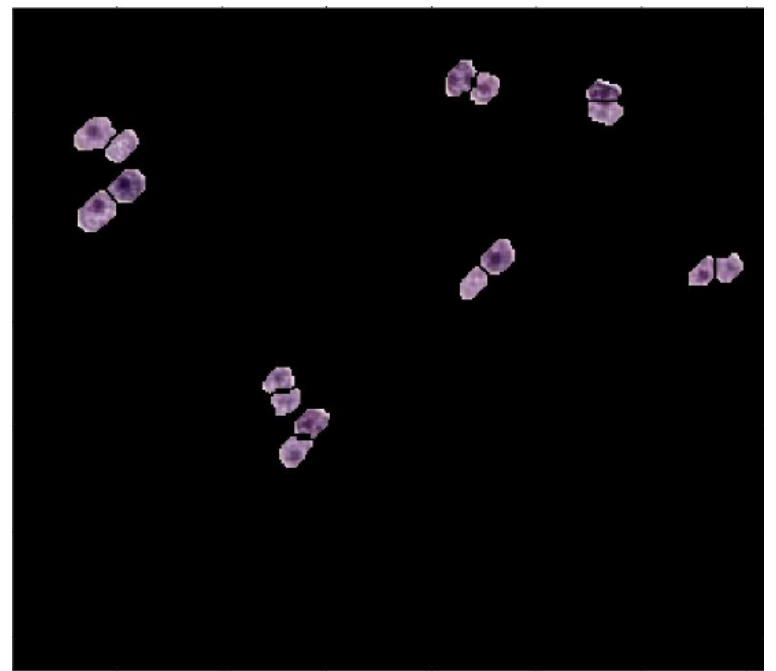

Figure. 9 Separated overlapped cells

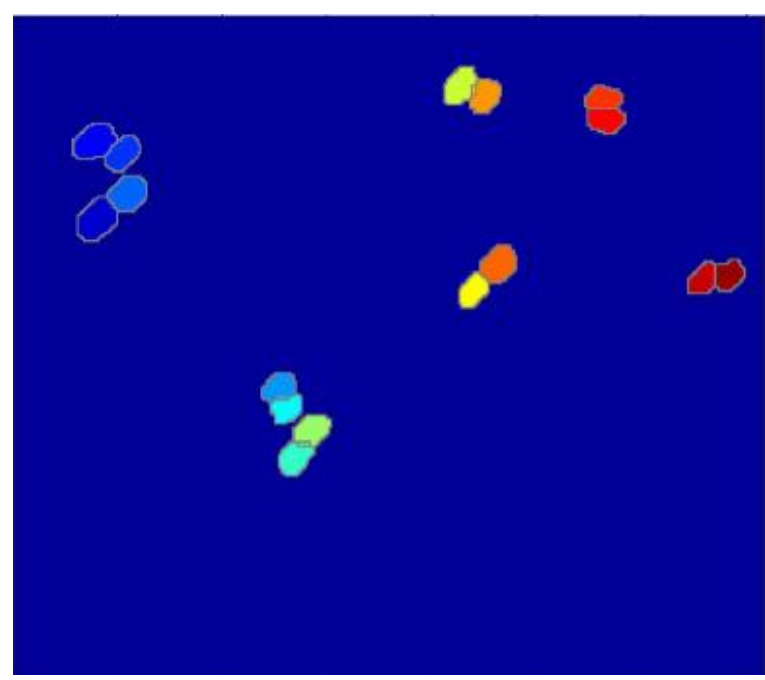

Figure. 10 Watershed method for cell separation

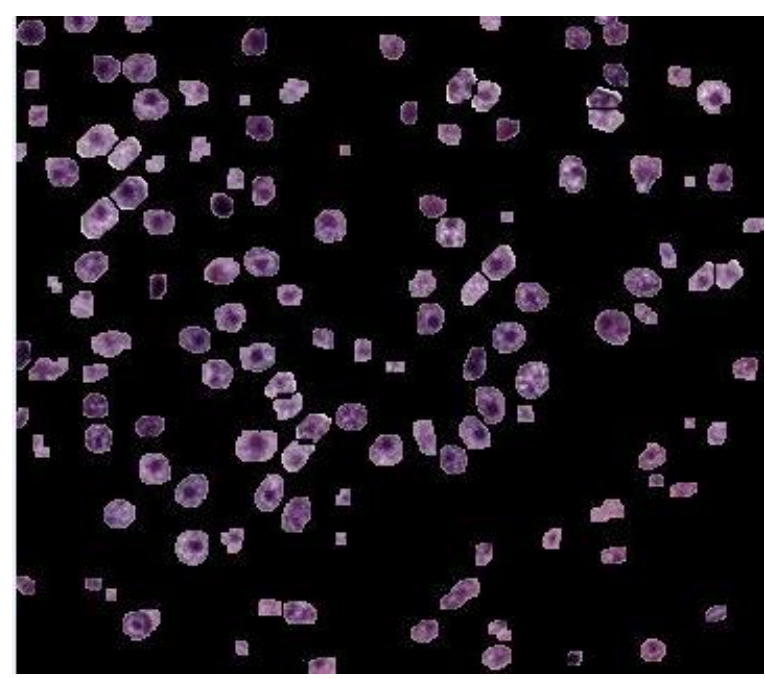

Figure. 11 Combined nuclei cells and non-nuclei cells

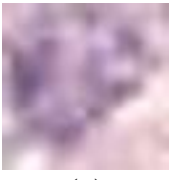

(a)

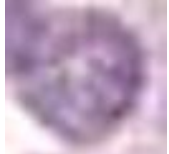

(b)

Figure. 12 OR-ACM image: (a) Non- nuclei cells and (b) Non- nuclei cells

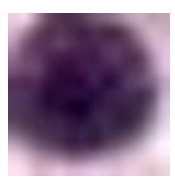

(a)

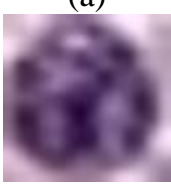

(b)

Figure. 13 OR-ACM image: (a) Non-affected nuclei cell and (b) Dilated nuclei cell

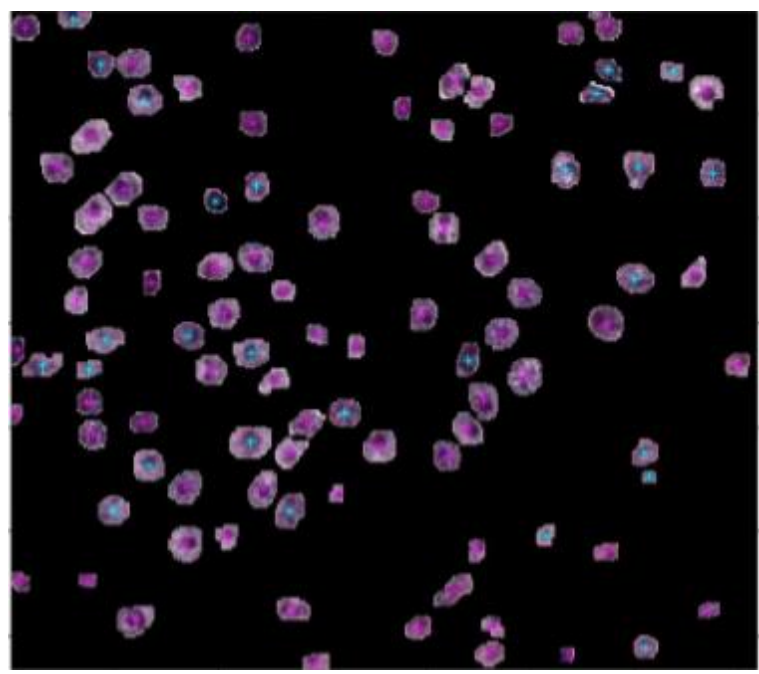

Figure. 14 Combined nuclei and non-nuclei cells

In Fig. 13, the nuclei cells are further classified into two groups such as Non-affected cells in Fig. 13 (a) and the affected or dilated cells in Fig. 13 (b). Differentiation between dilated nuclei cells and nonaffected nuclei cells are displayed in Fig. 14.

Here, the dilated cells are undertaken to classify into two levels of cells like micro level cells $(<2 \mathrm{~mm})$ and macro level cells $(2-3 \mathrm{~mm})$, which is symbolized in Fig. 15. Macro and micro nuclei cells are cropped from the dilated image for ease representation, which is mentioned in the Figs. 16 and 17 respectively. 


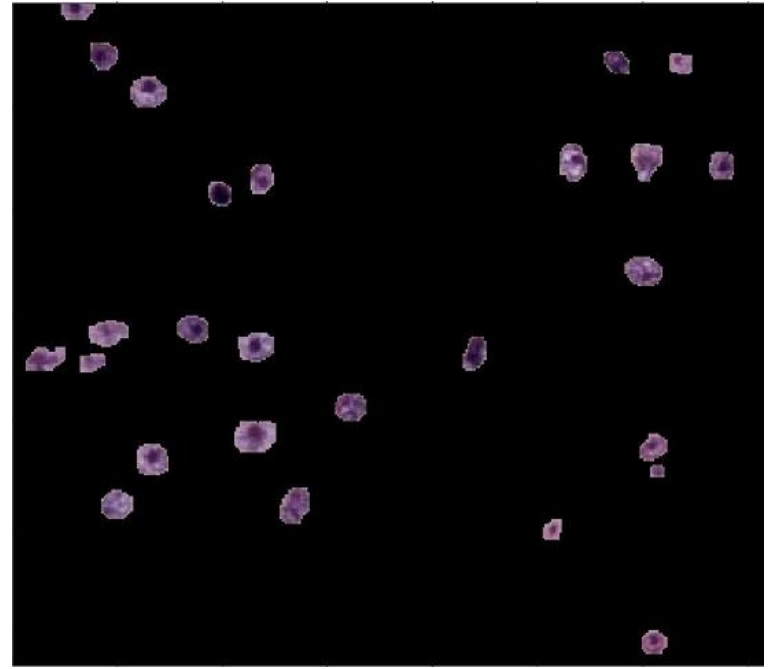

Figure. 15 Dilated nuclei cells

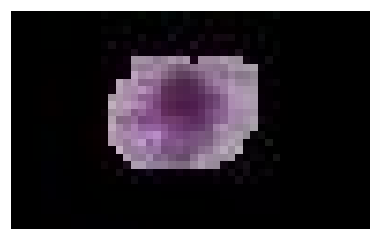

Figure. 16 Macro Dilated nuclei cells

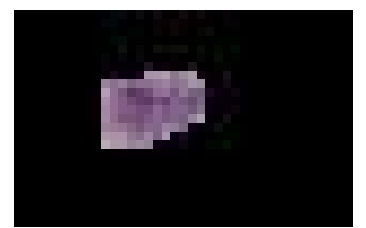

Figure. 17 Micro Dilated nuclei cells
Performance evaluation of existing methods and the proposed method: OR-ACM is compared in the Table.1, which depends on the accuracy of nonnuclei and nuclei cells recognition rate. D.S. Ashour, D.M. Abou Rayia, M. Maher Ata, A.S. Ashour, and M.M. Abd Elnaby, [16] developed a new hybrid combination of statistical features with Empirical Mode Decomposition (EMD). These combined features were further classified using Support Vector Machine (SVM). The developed approach achieved the classification accuracy of $80 \%$. N.S. Guptha, and K.K. Patil, [17] classifies the normal and cirrhotic cells with the help of AR-KFCM technique and distance between consecutive nuclei and non-nuclei were estimated by using earth mover's distance. This methodology achieved the classification accuracy of $86 \%$. Compared to these two existing methods, the proposed methodology achieved better result in terms of accuracy.

In ARKFCM, the recognition rate depends on distance measure. The methods implemented for determining the Nuclei and Non-nuclei recognition rate by Euclidean distance and earth mover's method. Hence, in OR-ACM, the recognition rate depends on the difference between fore ground and background area.

Table 2 compared the recognition rate of micro nuclei cells and macro nuclei cells for OR-ACM algorithm with-out watershed and with watershed algorithm. The proposed method: OR-ACM with watershed algorithm delivered better performance compared to existing approach.

Table 1. Performance evaluation between existing and proposed method (Nuclei and Non-nuclei cells)

\begin{tabular}{|c|c|c|c|}
\hline ALGORITHMS & $\begin{array}{c}\text { Non- Nuclei } \\
\text { Recognition rate (\%) }\end{array}$ & $\begin{array}{c}\text { Nuclei } \\
\text { Recognition rate (\%) }\end{array}$ & Average accuracy (\%) \\
\hline EMD-SVM [16] & 78 & 82 & 80 \\
\hline $\begin{array}{c}\text { ARKFCM - Euclidean } \\
\text { distance }\end{array}$ & 90 & 80 & 85 \\
\hline $\begin{array}{c}\text { ARKFCM- Earth movers } \\
\text { method [17] }\end{array}$ & 92 & 80 & 86.5 \\
\hline $\begin{array}{c}\text { OR-ACM (Without- } \\
\text { watershed) }\end{array}$ & 90 & 81 & 88 \\
\hline $\begin{array}{c}\text { Proposed OR-ACM (With- } \\
\text { watershed) }\end{array}$ & 94 & 82 & 86 \\
\hline
\end{tabular}

Table 2. Performance evaluation between existing and proposed method (Micro nuclei and Macro nuclei cells)

\begin{tabular}{|c|c|c|c|}
\hline ALGORITHMS & $\begin{array}{c}\text { Micro Nuclei } \\
\text { Recognition Rate (\%) }\end{array}$ & $\begin{array}{c}\text { Macro Nuclei } \\
\text { Recognition rate (\%) }\end{array}$ & $\begin{array}{c}\text { Average } \\
\text { accuracy (\%) }\end{array}$ \\
\hline OR-ACM (Without- watershed) & 76 & 80 & 78 \\
\hline $\begin{array}{c}\text { Proposed OR-ACM (With- } \\
\text { watershed) }\end{array}$ & 79 & 83 & 81 \\
\hline
\end{tabular}




\section{Conclusion}

This paper evaluates a modern approach (ORACM) for segmenting the nuclei and non-nuclei cells from the liver histopathology image. After segmenting, the nuclei cells are characterized into two levels such as macro nuclei and micro nuclei cells, which are evaluated based on semantic feature extraction (combination of both low and high level features) and classified by applying multi-class SVM classifier. Finally, the experimental outcome indicates that the advanced method shows $3 \%$ of improvement in macro and micro nuclei prediction in terms of accuracy than the existing schemes. In the future work, for further improving the classification accuracy, a hybrid clustering segmentation is performed with multi-objective classification.

\section{References}

[1] P.J. Brown, T.J. Whitbread, N.J. Bell, and F.A. Burden, "Hemosiderin deposition in Donkey (Equusasinus) liver: Comparison of liver histopathology with liver iron content", Research in Veterinary Science, Vol.90, No.2, pp.275-279, 2011.

[2] P. Ventura, G. Venturelli, M. Marcacci, M. Fiorini, S. Marchini, C. Cuoghi, and A. Pietrangelo, "Hyperhomocysteinemia and MTHFR C677T polymorphism in patients with portal vein thrombosis complicating liver cirrhosis", Thrombosis Research, Vol.141, pp.189-195, 2016.

[3] E.L. Zhang, Z.Y. Zhang, S.P. Wang, Z.Y. Xiao, J. Gu, M. Xiong, and Z.Y. Huang, "Predicting the severity of liver cirrhosis through clinical parameters", Journal of Surgical Research, Vol.204, No.2, pp.274-81 2016.

[4] H. Bhardwaj, B. Bhardwaj, and A. Awab, "Transudative chylothorax in a patient with liver cirrhosis: A rare association", Heart \& Lung: The Journal of Acute and Critical Care, Vol.44, No.4, pp.363-365, 2015.

[5] Y. Pu, S. Zhang, R. Zhou, N. Huang, H. Li, W. Wei, L. Li, C. Huang, J. Yang, Z. Li, “ IL-17A up-regulates expression of endothelial tissue factor in liver cirrhosis via the ROS/p38 signal pathway", Biochemical and Biophysical Research Communications, Vol.470, No.1, pp.41-7, 2016.

[6] R. Suganya, and S. Rajaram, "Content based image retrieval of ultrasound liver diseases based on hybrid approach", American Journal of Applied Sciences, Vol.9, No.6, pp.938, 2012.
[7] H. Su, F. Xing, and L. Yang, "Robust Cell Detection of Histopathological Brain Tumor Images Using Sparse Reconstruction and Adaptive Dictionary Selection", IEEE Transactions on Medical Imaging, Vol.35, No.6, pp.1575-1586, 2016.

[8] L.J. Binkowski, K. Sawicka-Kapusta, J. Szarek, E. Strzyżewska, and M. Felsmann, "Histopathology of liver and kidneys of wild living Mallards Anas platyrhynchos and Coots Fulica atra with considerable concentrations of lead and cadmium", Science of the Total Environment, Vol.450-451, pp.326-333, 2013.

[9] R.B. Allan and K.A. Thoirs, "A comparison of liver surface and hepatic vein wall ultrasound as markers for fibrosis or cirrhosis of the liver", Radiography, Vol.20, No.1, pp.8-14, 2014.

[10] V. de Lédinghen, V.W. Wong, J. Vergniol, G.L. Wong, J. Foucher, S.H. Chu, B. Le Bail, P.C. Choi, F. Chermak, K.K. Yiu, W. Merrouche, and H.L. Chan, "Diagnosis of liver fibrosis and cirrhosis using liver stiffness measurement: comparison between $\mathrm{M}$ and XL probe of Fibro Scan", Journal of Hepatology, Vol.56, No.4, pp.833-839, 2012.

[11] A. Chattopadhyay, S. Podder, S. Agarwal, and S. Bhattacharya, "Fluoride-induced histopathology and synthesis of stress protein in liver and kidney of mice", Archives of Toxicology, Vol.85, No.4, pp.327-335, 2011.

[12] S. Saleki, A.A. Ardalan, and A.J. Nejad, "Liver histopathology of fatal phosphine poisoning", Forensic Science International, Vol.166, No.2, pp.190-193, 2007.

[13] G.T. Brown and D.E. Kleiner, "Histopathology of nonalcoholic fatty liver disease and nonalcoholic steatohepatitis", Metabolism, Vol.65, No.8, pp.1080-1086, 2015.

[14] M. Ajdari and M.Z. Ghahnavieh, "Histopathology effects of nickel nanoparticles on lungs, liver, and spleen tissues in male mice", International Nano Letters, Vol.4, No.3, pp.1-4, 2014.

[15] I. Ashok, D. Wankhar, R. Sheeladevi, and W. Wankhar, "Long-term effect of aspartame on the liver antioxidant status and histopathology in Wistar albino rats", Biomedicine \& Preventive Nutrition, Vol.4, No.2, pp.299-305, 2014.

[16] D.S. Ashour, D.M. Abou Rayia, M. Maher Ata, A.S. Ashour, and M.M. Abd Elnaby, "Hybrid feature extraction techniques for microscopic hepatic fibrosis classification", Microscopy Research and Technique, 2018.

[17] N.S. Guptha and K.K. Patil, "Earth mover's distance-based CBIR using adaptive regularised 
Kernel fuzzy C-means method of liver cirrhosis histopathological segmentation", International Journal of Signal and Imaging Systems Engineering, Vol.10, No.1-2, pp.39-46, 2017. 